Vol. 13(1), pp. 1-7, 16 January, 2018

DOI: 10.5897/IJPS2017-4696

Article Number: E90B70F55617

ISSN 1992 - 1950

Copyright (C2018

International Journal of Physical

Author(s) retain the copyright of this article

Sciences

http://www.academicjournals.org/IJPS

\title{
A comparative study of maximum power point tracker approaches based on artificial neural network and fuzzy controllers
}

\author{
Moustapha Séne ${ }^{1}$, Fatou Ndiaye ${ }^{1}$, Marie E. Faye ${ }^{1}$, Saliou Diouf ${ }^{2}$ and \\ Amadou S. Maïga ${ }^{1^{*}}$ \\ ${ }^{1}$ Electronics IT Telecommunications and Renewable Energy Laboratory (LEITER), Gaston Berger University, \\ Route de Ngallele, P. O. Box 234 Saint-Louis, West Africa, Senegal. \\ ${ }^{2}$ Information Processing Laboratory (LTI), Higth Polytechnic School, Cheikh Anta Diop University, \\ P. O. Box 5085 Dakar-Fann, West Africa, Senegal.
}

Received 12 November, 2017; Accepted 20 December, 2017

\begin{abstract}
The performances of a photovoltaic (PV) module connected to a load through a conversion stage (chopper, inverter) are linked to the average electricity output including the delivered power. Nevertheless, the efficiency depends on atmospheric parameters as temperature, irradiance, and wind speed. To make electrical power available, Maximum Power Point Trackers (MPPT) algorithms are developed to keep up the PV module at optimal operating point with regard to climatic variations. This paper proposes an assessment of Artificial Neural Networks model based on MultiLayer Perceptron (MLP) and Radial Basis Function (RBF). A comparative study with an Adaptive Neuro-Fuzzy Inference System and a hybrid neural network RBF/MLP is done using measured data to optimize the maximum power point of a photovoltaic generator.
\end{abstract}

Key words: Multilayer perceptron, radial basis function, maximum power point trackers, neuro-fuzzy.

\section{INTRODUCTION}

The optimization of the power supply is a fundamental problem for electrical generator monitoring. Many improvements have been made on Maximum Power Point Tracker (MPPT) algorithms to control the DC/DC converter inserted on photo-voltaic plant (Elgendy et al., 2012; Mastromauro et al., 2012). Considered as the P\&O algorithm and the one of Incremental Conductance (Elgendy et al., 2016), electrical performances are of low reliability and output average, because the maximum power of photovoltaic (PV) module depends on the impedance of the load to which it is connected; but the major drawback is the perturbation due to changing atmospheric conditions (Zou et al., 2012).

Several experimental studies based on the Artificial Neural Network (ANN) method, have shown the variation of the electrical performances of the PV module, using a

*Corresponding author. E-mail: asmaiga1962@gmail.com.

Author(s) agree that this article remain permanently open access under the terms of the Creative Commons Attribution License 4.0 International License 


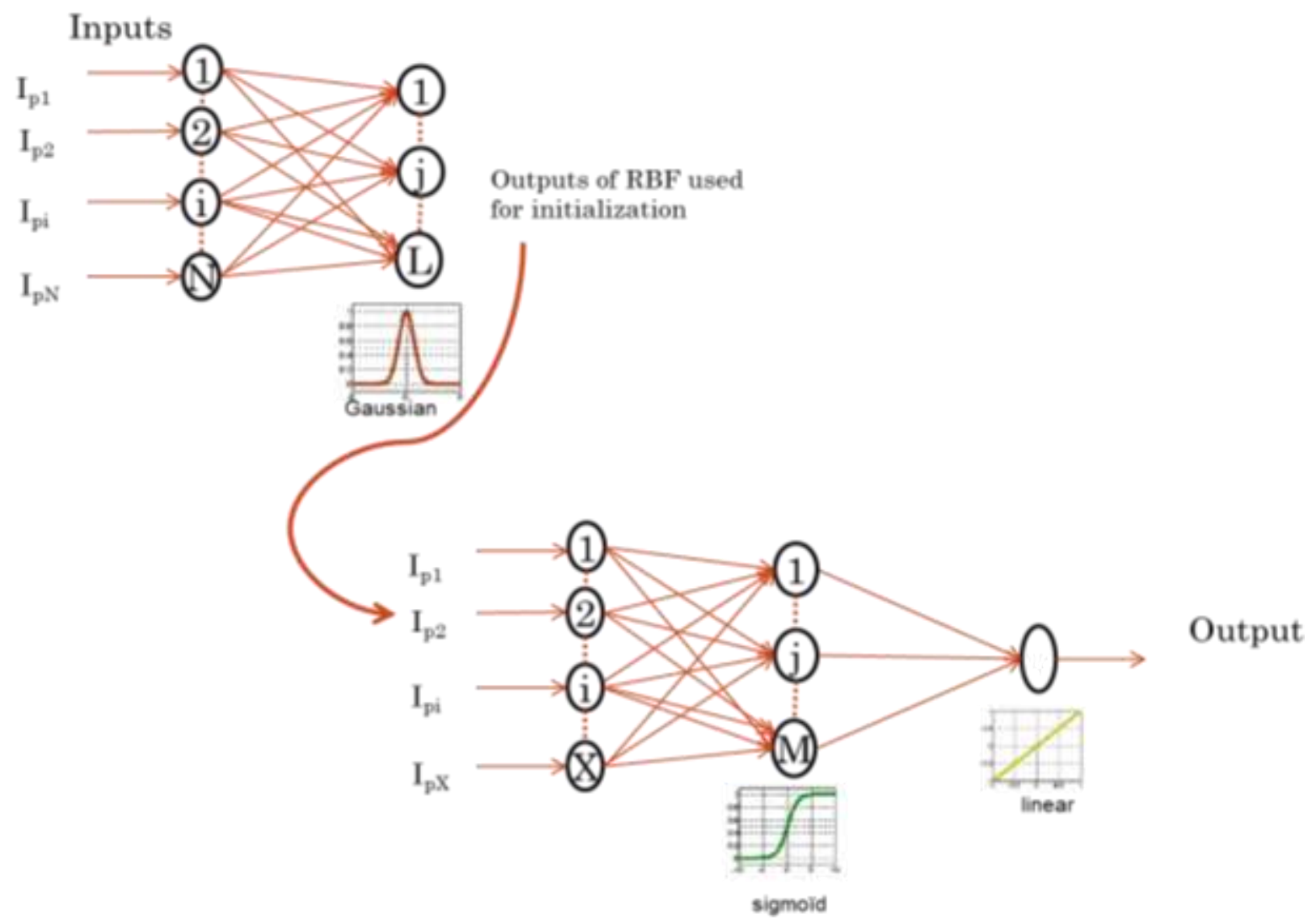

Figure 1. Architecture of the hybrid model.

database of measured irradiance, the temperature and also the optimum duty cycle (Saloux et al., 2011; Reisi et al., 2013). MultiLayer Perceptron (MLP) and Radial Basis Function (RBF) networks must be used to provide nonlinearity and self-adapting capability in controlled plants (Haskin, 2005).

This paper describes an assessment developed to provide different approaches for determining the maximum power of photovoltaic system in Sahelian region, where standard characteristics are not applicable. We present a comparison of MPPT approaches based on Artificial Neural Network (ANN) and Neuro-Fuzzy Controllers. It focuses on performances errors of the voltage curves relation between the measured electrical power and the developed model of a PV generation system.

\section{HYBRID ANN MODEL}

The MPPT strategy proposed here is based on a knowledge principle known as ANN which is an adaptive controller that can be developed for energy applications such as tracking the maximum power point of the PV module according to experimental data (Enany, 2017). In modern fields, a multilayer perception network trained by a supervised learning backpropagation algorithm associated to Levenberg-Marquardt method minimizes the mean square error layer (Ndiaye et al., 2015). A hybrid model has been developed in order to calculate the maximum power considering the changing atmospheric climate. The architecture of the mentioned model is composed of both RBF and MLP schemes used to explore the maximum power:

(i) The RBF scheme performs a local approach of the input vectors (temperature, wind speed and irradiance) to approximate the maximum current (Imax) and the maximum voltage (Vmax) delivered by a PV module.

(ii) The MLP scheme performs a comprehensive approach to the space of input vectors (Imax; Vmax) to derive the maximum power optimally (Bahgat et al., 2005; Elgharbi et al., 2012).

The architecture of this RBF/MLP hybrid model is described in Figure 1. The input layers contain three neurons as it composed of three inputs (solar radiation, ambient temperature and wind speed). The first hidden layer includes twenty neurons; this number is selected according to network stability and electrical parameters 
(Imax; Vmax). The second hidden layer (two neurons) and the third (twenty neurons) are used to perform the aforementioned electrical parameters with an orthogonal least square algorithm. The output layer contains one neuron used to find the maximum power. A mathematical function is applied to the last hidden layer to obtain the output value $a_{p j}$ corresponding to the input vector $I_{p i}$ :

$a_{p j}=f_{l}\left(\beta_{j}+\sum_{i=1}^{N} P_{i j} \cdot I_{p i}\right)$

Here, $f_{l}$ is the transfer function of hidden neuron which has a weight $P_{i j}$ from the input vector $I_{p i}$ and a bias $\beta_{j}$. Weights and biases are updated with a specified learning function.

\section{ADAPTIVE NEURO-FUZZY CONTROLLER}

The Adaptive Neuro-Fuzzy controller is a training algorithm for Takagi and Sugeno fuzzy inference systems (Vafaei et al., 2015). It is based on both learning algorithm as the least-squares method and the backpropagation gradient descent method used to emulate a given training input-output data set (Jang and Anfis, 1993, 1995). The FIS membership function parameters $X_{i}^{k}$ invoked an output node $i$ applied to a layer $k$ as the following:

$X_{i}^{k}=f\left(X_{1}^{k-1} \ldots X_{n_{k-1}}^{k-1}, a, b, c \ldots.\right)$

With $n_{k-1}$ the number of nodes in layer $k-1$ and $a, b, c$, $\ldots$ the $(i, k)$ node parameters. A typical fuzzy rule in a Takagi and Sugeno fuzzy model has the format:

If $x$ is $\mathrm{A}_{\mathrm{i}}$ and $\mathrm{y}$ is $\mathrm{B}_{\mathrm{i}}$ then $O_{i}=f_{i}(x, y)$.

Where $A_{i}$ and $B_{i}$ are fuzzy sets in the antecedent; $O_{i}=$ $f_{i}(x, y)$ takes usually a polynomial form:

$o_{i}=f_{i}(x, y)=a_{i} x_{i}+b_{i} y_{i}+c_{i}$

The proposed MPPT ANFIS controller uses 125 fuzzy rules related to three inputs variables which are mapped into a fuzzy inference system to a crisp output (maximum power). It should be noted that our objective is to evaluate the error performances regarding three terms: the MSE, the MAE and the correlation coefficient. Indeed, each climatic measure associated to the problem is fuzzified by defining a membership function that corresponds to Sugeno. Furthermore we need to describe the input variables using linguistic characterization and every linguistic variable can have five linguistic terms shown in Figure 2: $\{\mathrm{VL}, \mathrm{SL}, \mathrm{SH}, \mathrm{MH}$, $\mathrm{VH}$ \} (the training sets derived from these terms can be written in the form Very Lower, Small Lower, Small Higher, Medium Higher, Very Higher) and their membership functions are of the Gaussian form characterized by two parameters.

\section{EXPERIMENTAL PROTOCOL}

The experimental set-up protocol used for validation of the described controller chain is based on MPPT techniques for two typical days (cloudy and sunny). Table 1 gives the electrical characteristics of the AP100S5 PV module device and the experimental site geographical characteristics. So, three environmental inputs have been measured (in Niamey/Niger) to approximate the maximum power point: the irradiance, the ambient temperature and the wind speed corresponding to changing atmospheric conditions (typical days). A picolog manager is used providing the PV module outputs with electrical values following the current, the voltage and the power. The main problem of experimental protocol is to interpret the maximum power point within MPPT algorithm whatever the weather conditions. To insure adaptive controller, the latter is always adjusted to the trained model then compared to measured data set. In case of convergence, the routine controller is emulated by computing error performances (Figure 3).

\section{RESULTS AND DISCUSSION}

The adaptive controller is used exploiting ANN learning ability and fuzzy reasoning technique to follow the maximum power in PV devices. The resulting system is adjusted from available knowledge related to climatic data measured at Niamey NIGER. In order to compare the mentioned adaptive controllers, MPPT algorithms have been carried for typical given days (cloudy and sunny). It is interesting to note from Figure 4 that the PV module is performed to find the maximum power from typical sunny days' databases. Daily estimated data (Popthy), respectively measured data (Poptpv), are evaluated by computing error performances as a correlation coefficient (corr. Coef.), mean square error (MSE) and mean absolute error (MAE) for each controller. Table 2 summarizes results relative to obtained performances using adaptive RBF/MLP controller based scheme. The considered MSE and MAE errors performances decrease for involving cloudy days. This shows that adaptive RBF/MLP controller allows approximating maximum power point and must be adapted to abrupt climate changes.

Figure 5 shows the maximum power calculated with the adaptive neuro-fuzzy controller (Poptanf). This latter is compared to the computed RBF/MLP algorithm (Popthy) and the one of measured data (Poptpv) for typical atmospheric environments. For a sunny day covered from ANFIS controller, the errors performances are lower than the RBF/MLP. So inserting an MPPT Adaptive neuro-fuzzy controller allows the control of the power converter to provide maximum power to the load (Table 3). Concerning the typical cloudy days' data, ANFIS approach presents the higher error. This is due to the oscillation around the maximum power point. 


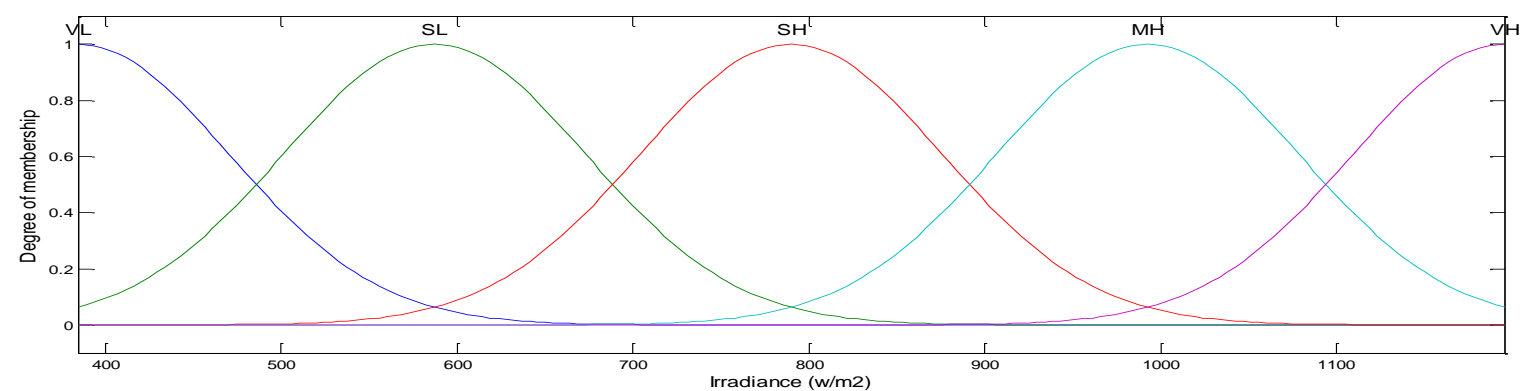

(a)

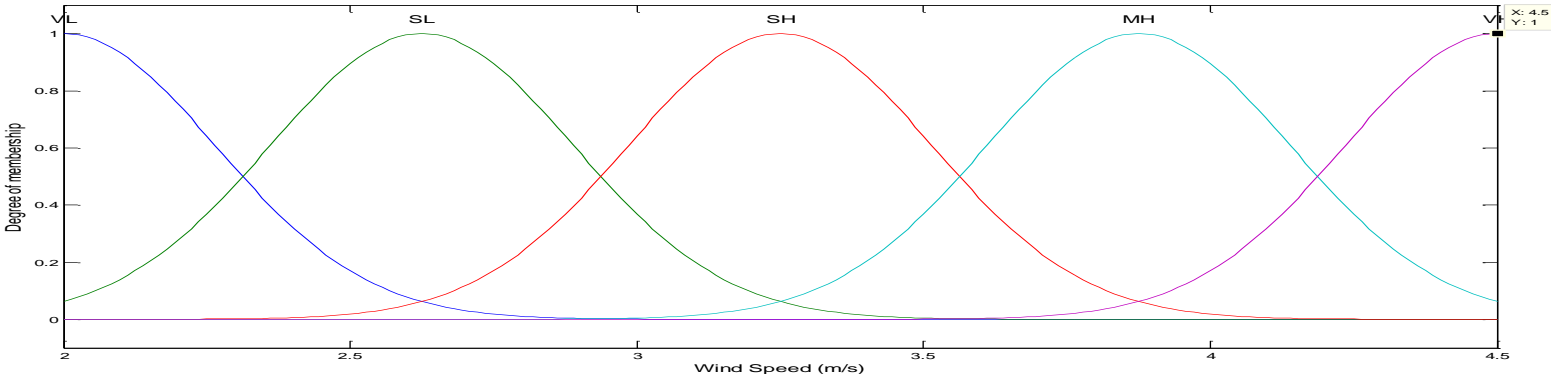

(b)

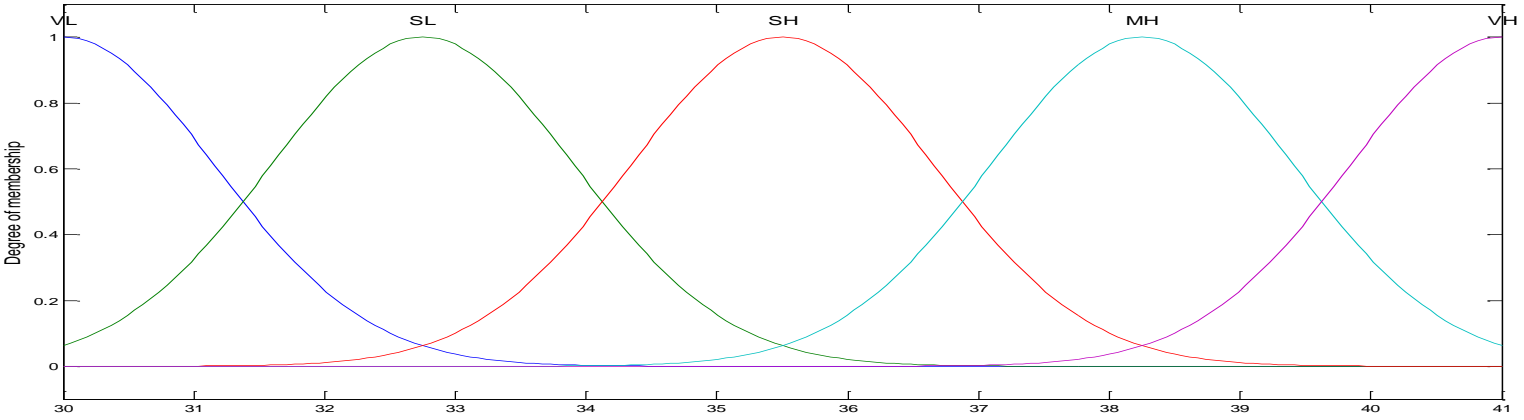

(c)

Figure 2. Linguistics terms issued typical sunny day (a) for the irradiance (b) for wind speed (c) for temperature.

Table 1. Electrical characteristics of AP100S5 PV module device and experimental site and geographical characteristics.

\begin{tabular}{lc}
\hline Parameter & Values \\
\hline Maximun power (Pm) & $70 \mathrm{~W}$ \\
Voltage at Pm (Vm) & $15.5 \mathrm{~V}$ \\
Current at Pm (Im) & $4.5 \mathrm{~A}$ \\
Open-circuit voltage (Voc) & $21.8 \mathrm{~V}$ \\
Short-circuit current (Isc) & $5.05 \mathrm{~A}$ \\
NOCT (Air $25^{\circ} \mathrm{C}$; sun $1 \mathrm{~kW} / \mathrm{m}^{2}$; wind $\left.1 \mathrm{~m} / \mathrm{s}\right)$ & $47 \pm 2^{\circ} \mathrm{C}$ \\
Temperature coefficient of Isc & $(0.065 \pm 0.015)^{\circ} /{ }^{\circ} \mathrm{C}$ \\
Temperature coefficient of Voc & $-(80 \pm 10) \mathrm{mV} /{ }^{\circ} \mathrm{C}$ \\
Temperature coefficient of power & $-(0.5 \pm 0.05) \% /{ }^{\circ} \mathrm{C}$ \\
Tolerance & \pm 3 \\
Solar Cell & $72 \mathrm{cells}$ \\
Number of cells in series & 36 \\
Number of cells in parallel & 2 \\
Strings Latitude (Niamey/Niger) & $13^{\circ} 31^{\prime} 01^{\prime \prime} \mathrm{North}$ \\
Longitude (Niamey/Niger) & $2^{\circ} 06^{\prime \prime} 00^{\prime \prime}$ East \\
Slant of PV module & 16 degrees South \\
\hline
\end{tabular}




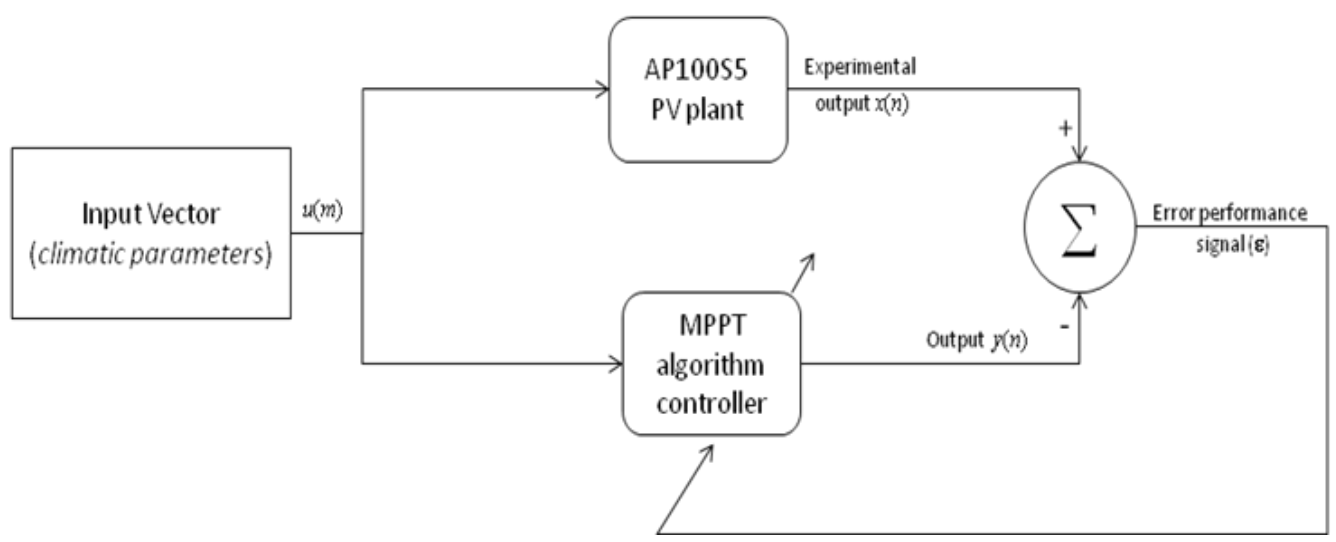

Figure 3. PV module adaptive controller chain.
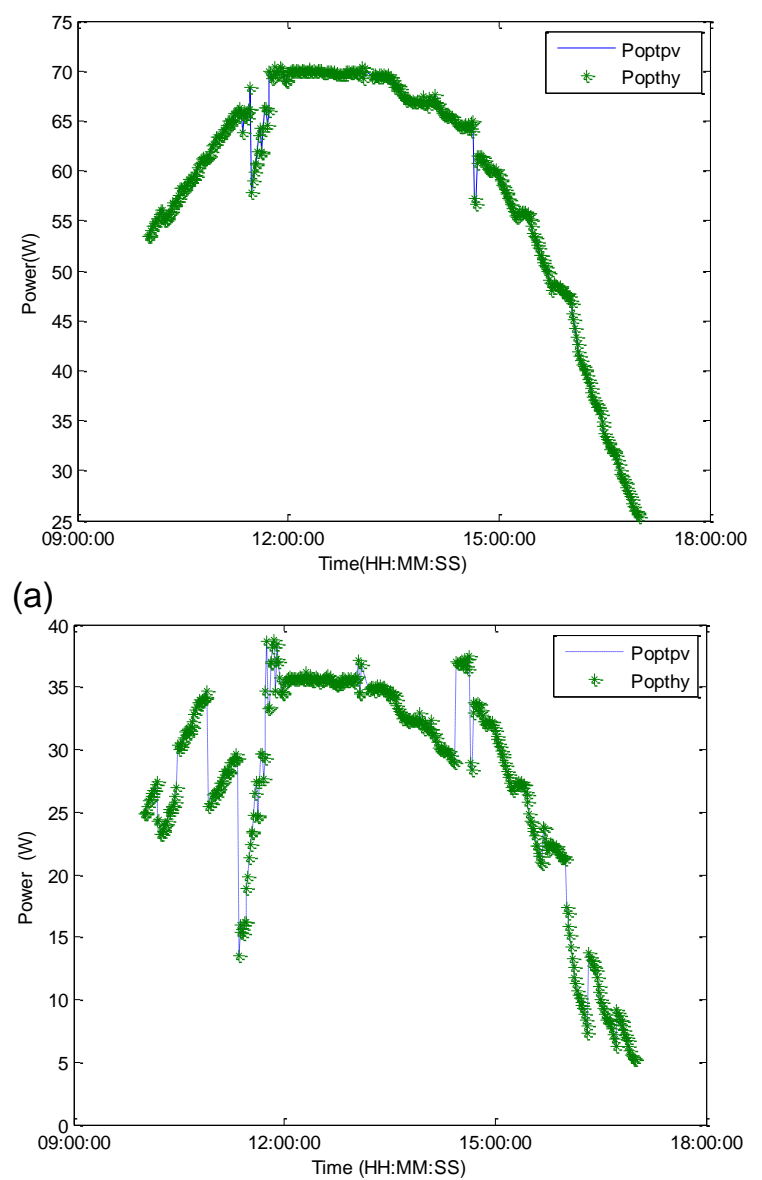

(b)

Figure 4. Maximum power issued adaptive RBF/MLP controller (a) sunny day; (b) cloudy day.

Table 2. Error performances of adaptive RBF/MLP controller.

\begin{tabular}{lccc}
\hline Day & MSE $(\mathbf{m W})$ & MAE $(\mathbf{m W})$ & corr. coef. \\
\hline Sunny day & 0.54 & 13.30 & 0.990 \\
Cloudy day & 0.17 & 00.40 & 0.999 \\
\hline
\end{tabular}




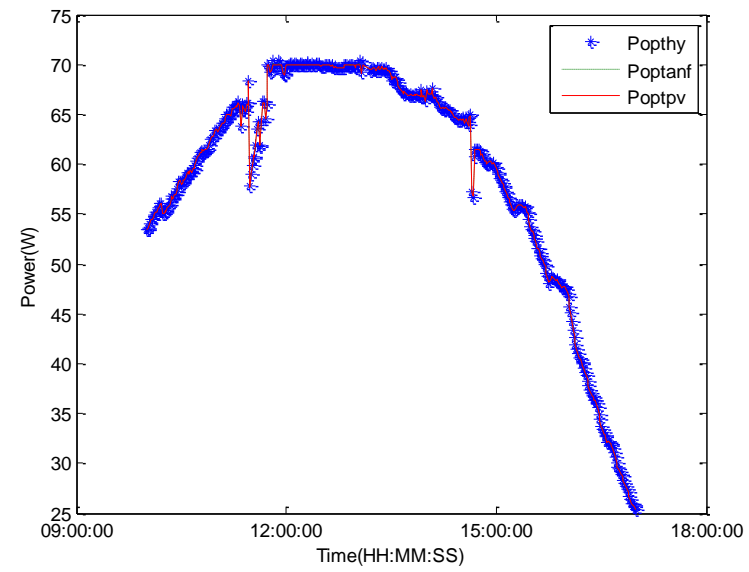

(a)

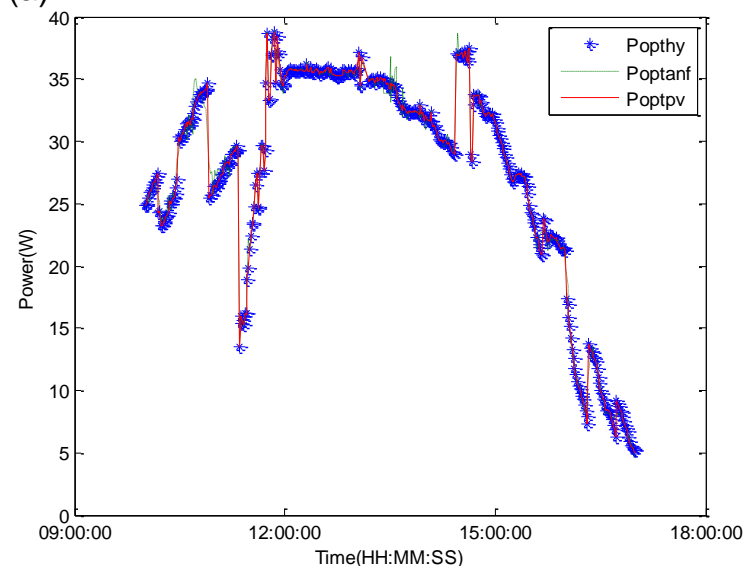

(b)

Figure 5. Maximum power issued adaptive neuro-fuzzy controller (a) sunny day; (b) cloudy day.

Table 3. Error performances of adaptive neuro-fuzzy controller.

\begin{tabular}{lccc}
\hline Day & MSE $(\mathbf{m W})$ & MAE $(\mathbf{m W})$ & corr. coef. \\
\hline Sunny day & 0.49 & 6.20 & 0.99 \\
Cloudy day & 4.36 & 3.72 & 0.997 \\
\hline
\end{tabular}

\section{Conclusion}

In photovoltaic plants, the operating point is important in terms of controlling the DC/DC converter. The right tracking for the maximum power of the PV generator will adapt to the impedance of the load to which it is connected. In order to predict the optimal power point, different controllers are used for tuning parameters of adaptive algorithms. In this study, maximum power points are tracked using adaptive algorithms tuned by climatic database. Results indicate improved performances between measured data and predicted ones of sunny days with the neuro-fuzzy model. Further studies involve hybrid ANN model used in sudden changes of climatic parameters which suit the aforementioned Sahelian region.

\section{CONFLICT OF INTERESTS}

The authors have not declared any conflict of interests.

\section{REFERENCES}

Bahgat ABG, Helwa NH, Ahmad GE, El Shenawy ET (2005). Maximum power point tracking controller for PV systems using neural networks. 
Renew. Energy 30:1257-1265.

Elgendy MA, Atkinson DJ, Zahawi B (2016). Experimental investigation of the incremental conductance maximum power point tracking algorithm at high perturbation rates. IET Renew. Power Gen. 10(2):133-139.

Elgendy MA, Zahawi B, Atkinson DJ (2012). Assessment of Perturb and Observe MPPT Algorithm Implementation Techniques for PV Pumping Applications. IEEE Trans. Sustain. Energy 3(1):21-34.

Elgharbi A, Mezghani D, Mami A (2012). A Maximum power point tracking method based on artificial neural network for a PV system. Int. J. Adv. Eng. Technol. IJAET.

Enany MA (2017). Cuckoo search-based maximum power point tracking controller for PV water pumping system. J. Renew. Sustain. Energy 9:063501.

Haskin S (2005). Neural networks: A comprehensive foundation. San Francisco Pearson Education.

Jang JS, Anfis R (1993). Adaptive-Network-based Fuzzy Inference System. IEEE Trans. Syst. Man Cybernetics 23:665-685.

Jang JS, Anfis R (1995). Neuro-Fuzzy Modeling and Control. Proc. IEEE 83(3):378-406.

Mastromauro RA, Liserre M, Dell'Aquila A (2012). Control Issues in Single Stage Photovoltaic Systems: MPPT, Current and Voltage Control. IEEE Trans. Ind. Inf. 8(2):241-254.

Ndiaye F, Sène M, Beye M, Seidou Maiga A (2015). Effects of climatic conditions on a polycrystalline photovoltaic module in Niger. Int. Lett. Chem. Phys. Astron. 55:60-66.
Reisi AR, Moradi MH, Jamasb S (2013). Classification and comparison of maximum power point tracking techniques for photovoltaic system: A review. J. Renew. Sust. Energy Rev. 19:433-443.

Saloux E, Teyessdou A, Sorin M (2011). Explicit model of photovoltaic panel to determine voltages and current at the maximum power point. Solar energy 85:713-722.

Vafaei S, Rezvani A, Gandomkar M, Izadbakhsh M (2015). Enhancement of grid-connected photovoltaic system using ANFISGA under different circumstances. Front. Energy 9(3):322-334.

Zou Y, Yu Y, Zhang Y, Luc J (2012). MPPT Control for PV Generation System Based on an Improved Inccond Algorithm. Elsevier, Int. Workshop Info. Electron. Eng. 105-109. 